\title{
Neuronal extracellular vesicle derived miR-98 prevents salvageable neurons from microglial phagocytosis in acute ischemic stroke
}

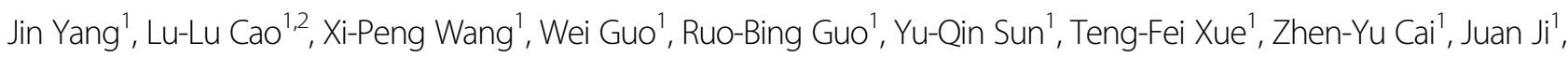
Hong Cheng ${ }^{3}$ and Xiu-Lan Sun ${ }^{1,4}$

\begin{abstract}
Extracellular vesicles (EVs), as a novel intercellular communication carrier transferring cargo microRNAs (miRNAs), could play important roles in the brain remodeling process after ischemic stroke. However, the detailed mechanisms involved in EVs derived miRNAs-mediated cellular interactions in the brain remain unclear. Several studies indicated that microRNA-98 (miR-98) might participate in the pathogenesis of ischemic stroke. Here, we showed that expression of miR-98 in penumbra field kept up on the first day but dropped sharply on the 3rd day after ischemic stroke in rats, indicating that miR-98 could function as an endogenous protective factor post-ischemia. Overexpression of miR-98 targeted inhibiting platelet activating factor receptor-mediated microglial phagocytosis to attenuate neuronal death. Furthermore, we showed that neurons transferred miR-98 to microglia via EVs secretion after ischemic stroke, to prevent the stress-but-viable neurons from microglial phagocytosis. Therefore, we reveal that EVs derived miR-98 act as an intercellular signal mediating neurons and microglia communication during the brain remodeling after ischemic stroke. The present work provides a novel insight into the roles of EVs in the stroke pathogenesis and a new EVs-miRNAs-based therapeutic strategy for stroke.
\end{abstract}

\section{Introduction}

Ischemic stroke, one of the most common disabling diseases, is mainly characterized by thromboembolic occlusion of the main artery supplying the brain. Clinically, rapidly recanalizing the occluded blood vessels and early re-establishing blood flow and tissue reperfusion could save part patients, who are eligible for thrombolysis and also expected to have the potential to improve functional outcome in the prognosis ${ }^{1-3}$. After stroke, the ischemic brain induces highly dynamic alterations, interacting processes and limited remodeling of the neurovascular unit, involving dysfunction of endothelial cells,

Correspondence: Xiu-Lan Sun (xiulans@njmu.edu.cn)

'Department of Pharmacology, Neuroprotective Drug Discovery Key

Laboratory, Jiangsu Key Laboratory of Neurodegeneration, Center for Global Health, Nanjing Medical University, Nanjing, China

${ }^{2}$ Zhongda Hospital, Southeast University, Nanjing, China

Full list of author information is available at the end of the article

Edited by A. Verkhratsky neurons, glia, pericytes, and associated tissues proteins ${ }^{4}$. When neurons suffer threatens from ischemic stimuli, they not only activate self-preservation but also release extracellular "help me" signals to adjacent cells and induce them to shift into beneficial phenotypes, to limit damage ${ }^{5}$. Some findings indicate that endangered or damaged neurons can release a repertoire of signaling molecules to instruct surrounding cells including microglia, control microglial function, and regulate microgliamediated phagocytosis and neuroprotection ${ }^{6}$. However, the signals involved in regulating the interactions between neurons and microglia remain unclear.

Currently, EVs grasp our eyes as a flexible medium in cell-to-cell interaction ${ }^{7-9}$. EVs, $<150 \mathrm{~nm}$ in diameter, are secreted into the extracellular space and then fused to neighboring cells to release their contents ${ }^{10,11}$. Emerging evidence suggests that EVs play a significant role in intercellular communication by transferring protein and

\section{(c) The Author(s) 2021}

(c) (i) Open Access This article is licensed under a Creative Commons Attribution 4.0 International License, which permits use, sharing, adaptation, distribution and reproduction cc) in any medium or format, as long as you give appropriate credit to the original author(s) and the source, provide a link to the Creative Commons license, and indicate if changes were made. The images or other third party material in this article are included in the article's Creative Commons license, unless indicated otherwise in a credit line to the material. If material is not included in the article's Creative Commons license and your intended use is not permitted by statutory regulation or exceeds the permitted use, you will need to obtain permission directly from the copyright holder. To view a copy of this license, visit http://creativecommons.org/licenses/by/4.0/. 
RNA cargo among components of the neurovascular unit after stroke ${ }^{12}$. Some researches indicate that EVs derived miRNAs play important roles in brain repair processes after stroke ${ }^{13-15}$. Surprisingly, the ratio of microRNAs (miRNAs) over total RNAs in EVs is higher than that in their parent cells ${ }^{7,16}$. MicroRNA-98 (miR-98), belonging to highly-conserved miRNAs, the let-7 group, has been reported to participate in the protection of blood-brain barrier function and the pathogenesis of ischemic stroke $^{17,18}$. One study showed that only miR-98 was significant decreased in the cultured hippocampal neurons at $1 \mathrm{~h}$ post oxygen-glucose deprivation (OGD) $30 \mathrm{~min}$, different from other miRNAs ${ }^{19}$, which indicated a specific role of miR-98 in stroke.

As is known to all, the brain is an "immune-privileged" organ, meaning that brain immunity is under tight control to protect nervous system from potential harmful immune reactions ${ }^{5,20}$. Microglia, the native immune cell in the central nervous system (CNS), once activated, exert key immune regulative function during ischemic stroke ${ }^{21-23}$. Accumulating evidence indicates that neurons could control microglial activation and influence microglial function, and then in turn microglia affect the neuronal survival during the ischemic stroke ${ }^{1}$. Microglia are the professional phagocytes in the brain and microglial phagocytosis is a double-edged sword ${ }^{24}$. Phagocytosis of dead neurons and neuronal debris is in part beneficial because it reduces inflammation ${ }^{25}$. However, they also engulf those dying or damaged salvageable neurons in the ischemic penumbra after ischemic stroke. Blocking the process of phagocytosis of stress-but-viable neurons is considered as a beneficial therapeutic strategy for ischemic stroke ${ }^{26-31}$.

Based on the above evidence, we hypothesize that neurons might release EVs containing miR-98, as a "help me" signal, to regulate microglial phagocytosis after ischemic stroke. The present work was to reveal the roles and the involved mechanisms of EVs derived miR-98 delivered from neurons to microglia in ischemic stroke.

\section{Results}

MiR-98 participates in the acute phase of ischemic stroke

First, in order to assess the clinical significance of miR98 in ischemic stroke, we determined the expression level of miR-98 in serum from 10 randomly selected acute ischemic stroke patients enrolled in hospital and corresponding non-stroke healthy controls (Supplementary Table 1). We found that miR-98 expression level was obviously downregulated in the serum of acute ischemic stroke patients compared with healthy controls and there were no significant differences between males and females (Fig. 1a, b). In the ischemic stroke rat models induced by transient middle cerebral artery occlusion (tMCAO) (assessed by laser doppler flowmetry, Supplementary Fig. 1), the levels of miR-98 in the serum were also decreased compared with sham group (Fig. 1c). The above results indicated the potential role of miR-98 in the ischemic stroke.

To further determine the function of miR-98 in the critical period of ischemic stroke pathogenesis, we quantified temporal changes of miR-98 expression of ischemic stroke rats within 1 day to 5 weeks after stroke using RT-qPCR (Fig. 1d). The expression levels of miR-98 markedly decreased in the core zone of ischemic brain on the 1st day and 3rd day, and rose during the subsequent observation period. Notably, miR-98 expression maintained the same level as the sham groups in the penumbra field on the first day but dropped sharply on the third day after stroke (Fig. 1e). The same alterations were confirmed by staining results examined by fluorescence in situ hybridization (FISH) (Fig. 1f-h).

Next, we validated the findings in the primary cultured neurons from rat cortex treated with 30-min OGD and reoxygenation. We determined the early temporal alterations of miR-98 level at 30 minutes, $1 \mathrm{~h}, 3 \mathrm{~h}, 5 \mathrm{~h}$ after reoxygenation with the starvation treatment for control (Fig. 1i). Interestingly, we found that the expression level of neuronal miR-98 showed an increase and then dropped both in starvation groups and in OGD-treated groups. What's more, we found miR-98 expression peaked at $3 \mathrm{~h}$ after reoxygenation and decreased markedly at $5 \mathrm{~h}$ after reoxygenation (Fig. 1j). These results suggest that miR-98 is involved in progression of ischemic stroke and might function as an endogenous protective factor in the early occurrence of ischemic stroke.

\section{MiR-98 can be packed into EVs transferred from neurons to microglia}

First, we collected primary neuronal-conditional media (NCM) from neurons (Fig. 2b, MAP2) which were treated with OGD $30 \mathrm{~min}$ and reoxygenation $3 \mathrm{~h}$, when neuronal miR-98 expression peaked (Fig. 2a). The collected NCM were added into primary cultured microglia (Fig. 2b, IBA1) and miR-98 in microglia was examined after $24 \mathrm{~h}$. We found miR-98 expression of microglia was significantly increased in microglia added NCM from neurons treated with OGD-R and has an increase trend $(p=0.177)$ in microglia added NCM from starvation treated neurons (Fig. 2c). We also demonstrated that miR-98 was expressed almost exclusively in neurons compared with microglia, using miR-98 expression of neuro-2a cells $(\mathrm{N} 2 \mathrm{a})$ as a baseline control (Fig. 2d). To verify the speculation that neuronal miR-98 could be packed into EVs and delivered from neurons to microglia, GW4869, a neutral sphingomyelinase inhibitor, was used to block EV secretion ${ }^{32}$. Interestingly, we found GW4869 $(20 \mu \mathrm{M})$ could reverse the increase of miR-98 expression level in microglia after added NCM from neurons treated with starvation and OGD/R (Fig. 2c). These results suggested that neuronal miR-98 


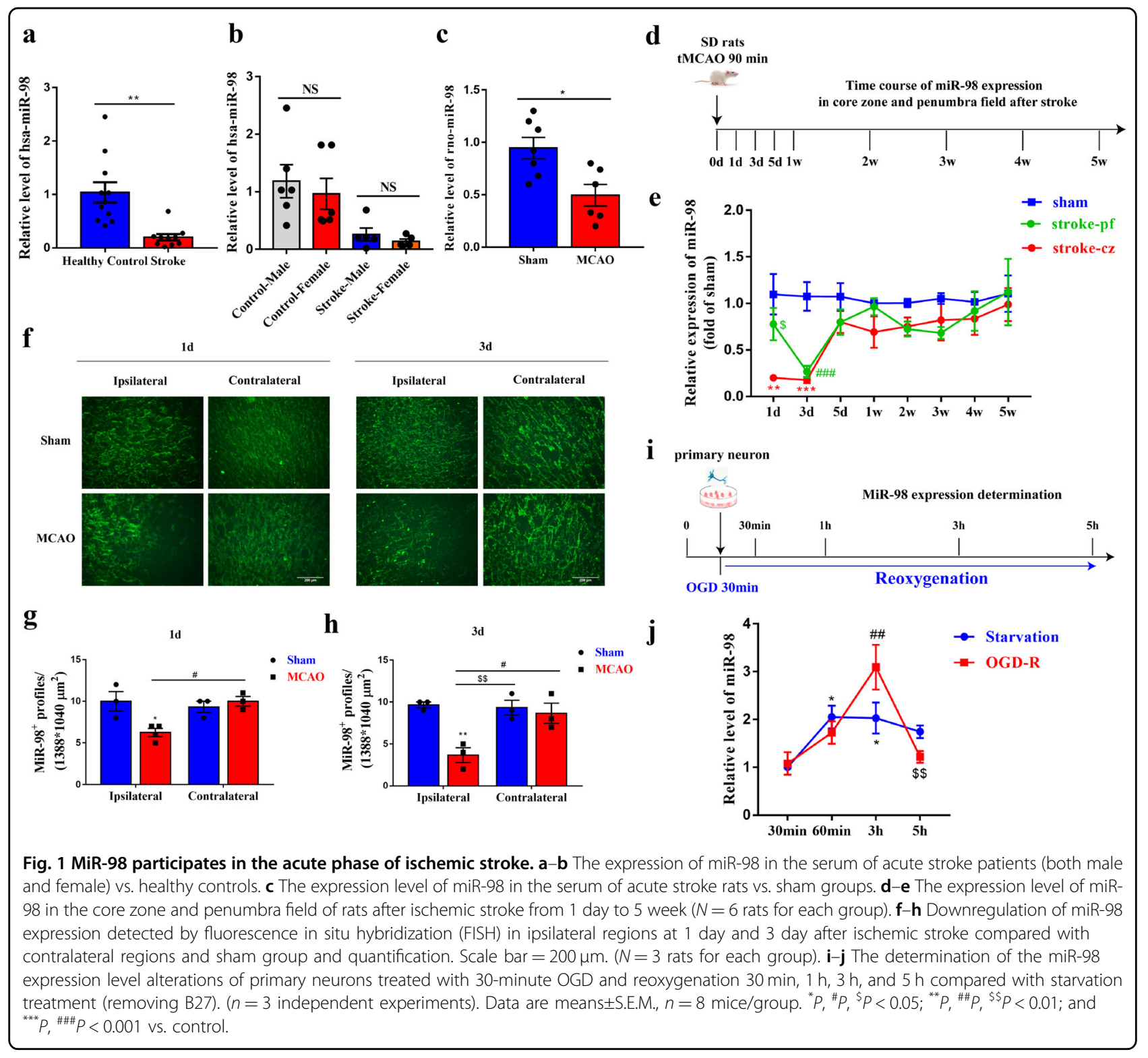

could be transferred via EVs secretion. Next, we isolated EVs from NCM and performed subsequent experiments (Fig. 2e). Transmission electron microscope (TEM) and Zetasizer Nano-ZS90 measurement identified the shape of "cup" with bilayer and size distribution of EVs (Zeta potential: $-14.1 \mathrm{mv}$; Average size: $71.42 \mathrm{~nm}$; polydispersity index: 0.410) (Fig. 2f, g). Western blotting analysis indicated that EVs markers ALIX and TSG101 were abundant in our extracted EVs without the endoplasmic reticulum marker Calnexin (Fig. 2h). Further, the yellow-orange fluorescent dye PKH26-labeled neuronal EVs were incubated with microglia labeled by CellTracker ${ }^{\mathrm{TM}}$ Green CMTPX Dye for $12 \mathrm{~h}$. We observed PKH26-labeled neuronal EVs internalized into the microglia (Fig. 2i). Moreover, internalization of neuronal EVs significantly increased the miR-98 expression in microglia compared with phosphate-buffered solution (PBS) treated control groups (Fig. 2j) after $24 \mathrm{~h}$ incubation. Altogether, these results reveal that miR-98 could be transferred from neurons to microglia via EVs secretion. In addition, we measured the serum levels of EVs miR-98 in both individuals and rats, which were also characterized by TEM (Fig. 2k, l, m, m), size distribution (Fig. 2o, p), and western blotting (Fig. 2q). We found that the serum levels of EVs miR-98 were decreased in both individuals and rats likewise.

\section{In vivo transfer of miR-98 into microglia through neuron- secreted EVs after ischemic stroke}

To further confirm whether neurons could transfer miR-98 to microglia via EVs secretion, the mature neuron-targeted 

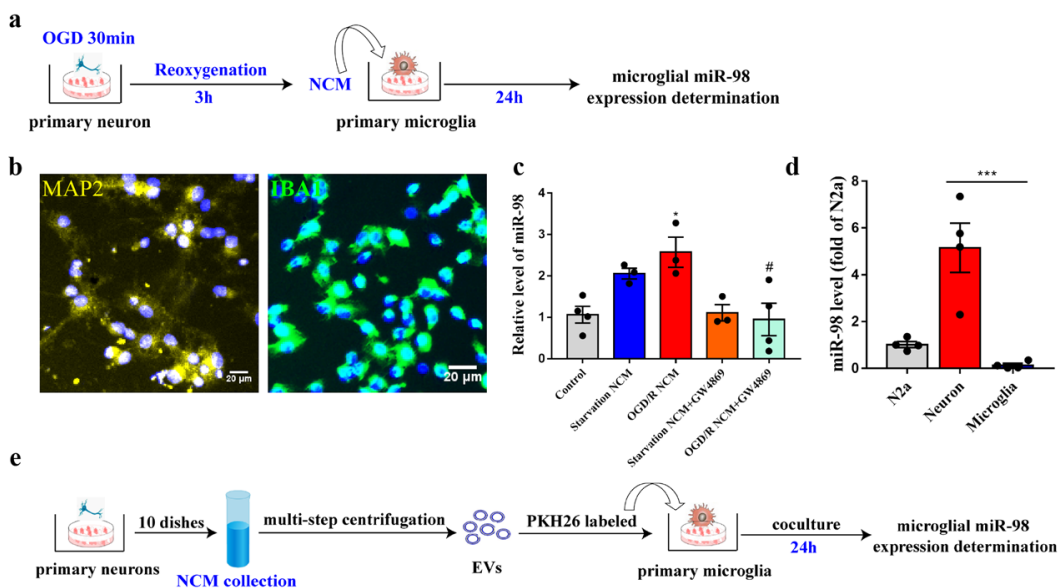

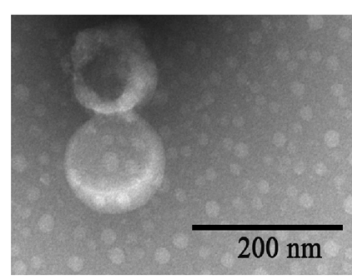

h
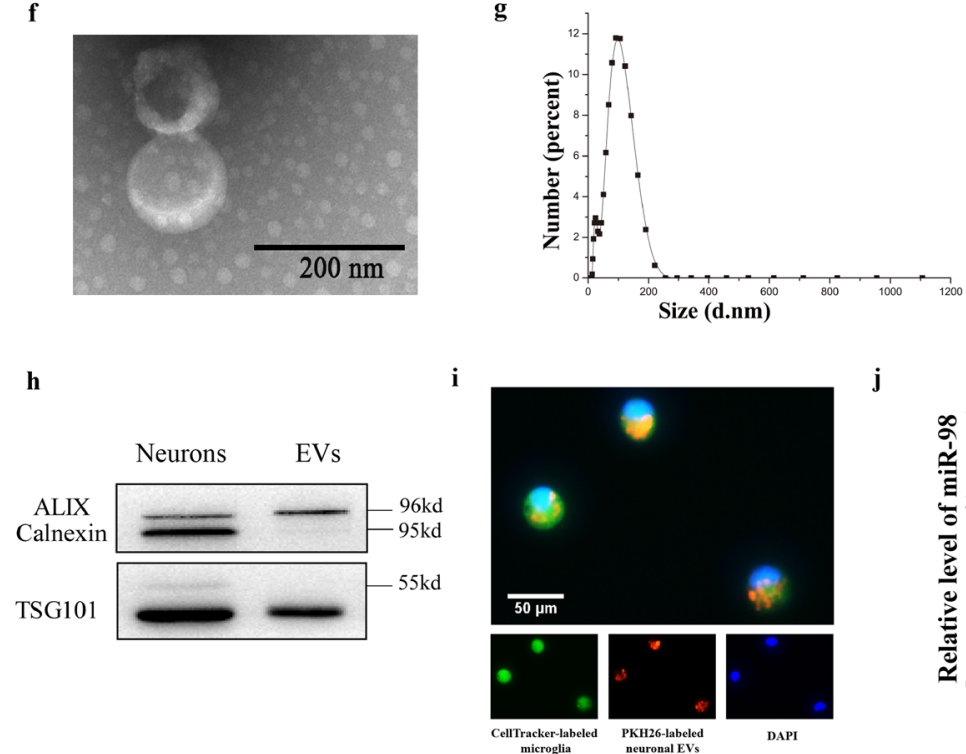

DAPI
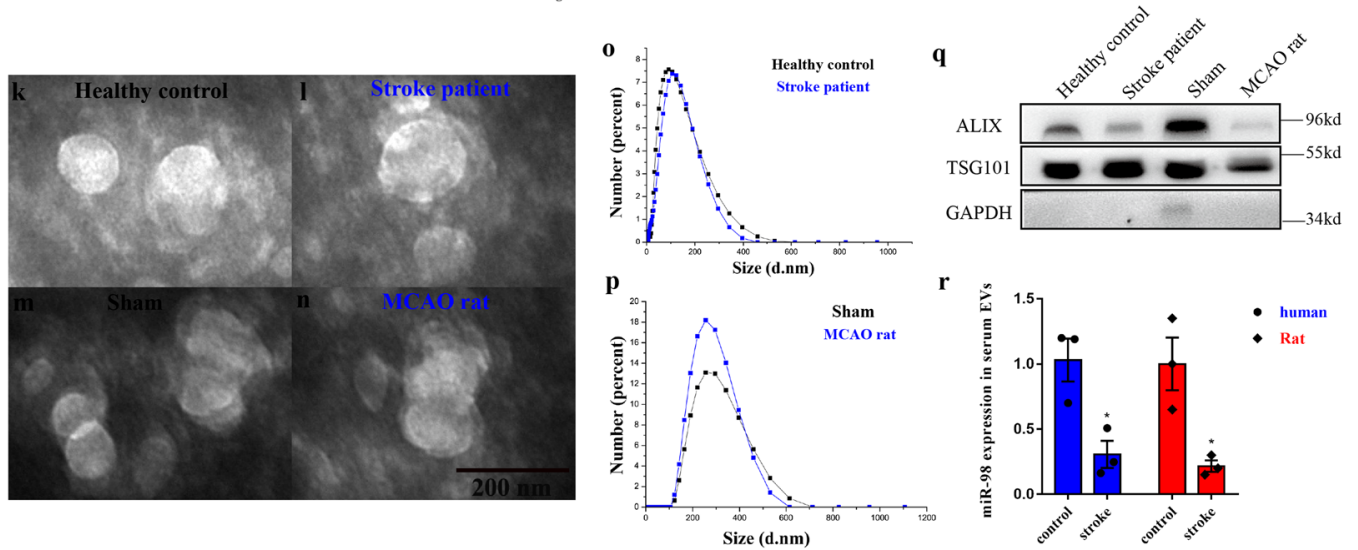

Fig. 2 (See legend on next page.)

adeno-associated virus (AAV, SYN promoter, AAV2/9) was used to label neuronal EVs. A previous study reported that CD63 could be applied to label EVs ${ }^{33}$. Based on this, we injected the viruses pAAV-SYN-CD63-mCherry3FLAG combined with pAAV-SYN-EYFP-3FLAG-miR98 into the cortex of p20 C57BL/6 J mice (Fig. 3c), to label 
(see figure on previous page)

Fig. 2 MiR-98 packed into EVs can be transferred from neurons to microglia. a The procedure of primary neuronal-conditional media (NCM) collection and coculture with primary microglia. b Positive rate of Rat primary cortical neuron (MAP2) and microglia (IBA1). c The relative miR-98 expression level of microglia after added with NCM from starvation and OGD-R treatment and GW4869 blocking. ( $n=3$ independent experiments). d The relative miR-98 expression level between neuron and microglia of purification culture, using the miR-98 expression of Neuro-2a (N2a) cells as a baseline. e The procedure of extraction of primary neuronal EVs and co-cultured with microglia. $\mathbf{f}$ Transmission electron micrograph (TEM) of neuronal EVs. g Size distribution of neuronal EVs based on Zetasizer Nano-ZS90 measurements. $\mathbf{h}$ Western blot for EVs markers Alix, TSG101, and Calnexin. i Representative images of internalization of PKH26-labeled neuronal EVs into the CellTracker ${ }^{\mathrm{TM}}$ Green-labeled microglia $12 \mathrm{~h}$ after coculture. j Relative miR-98 level in microglia $24 \mathrm{~h}$ after incubated with PBS and neuronal EVs (extraction by $\sim 80 \sim 100 \mathrm{ml}$ neuronal media). ( $n=5$ independent experiments). Data are shown as means \pm S.E.M., ${ }^{*} P,{ }^{\#} P<0.05 ;{ }^{* *} P<0.01 ;{ }^{* * *} P<0.001$ vs corresponding control. k-n Transmission electron micrograph (TEM) of serum EVs from both individuals and rats. o- $\mathbf{p}$ Size distribution of serum EVs from both individuals and rats. $\mathbf{q}$ Western blot for EVs markers Alix, TSG101, and GAPDH. $\mathbf{r}$ The relative miR-98 expression level between in serum EVs between both individuals and rats.

the neuronal EVs and miR-98, respectively. pAAV-SYNMCS-EYFP-3FLAG was used as control label (Fig. 3a). We identified the infection region and efficiency of virus injection with immunofluorescence staining and the 3D reconstructions of Z-stack images (Fig. 3b). The colocalization of CD63-mCherry and miR-98-EYFP showed that these neurons expressed miR-98 (Fig. 3d). Three weeks after virus injection, the mice were exposed to $\mathrm{TMCAO}$ to establish the ischemic stroke model. After reperfusion $6 \mathrm{~h}$, we found that CD63-labeled and miR-98-EYFP-labeled neuronal EVs appeared outside of the neurons and internalized into IBA1-labeled microglia in the ipsilateral regions obviously (Fig. 3e). Very clearly, we observed the colocalization of CD63-labeled neuronal EVs, neuronal miR-98 and microglia (Fig. 3f, g) with Z-stack images and $3 \mathrm{D}$ reconstructions of the white dotted regions acquired by a two-photon microscope, which could be observed $3 \mathrm{~h}$ after reperfusion (Supplementary 3a-c). Therefore, we confirm that neurons can transfer miR-98 to microglia via EVs secretion after ischemic stroke.

\section{Overexpression of miR-98 can prevent stressed but salvageable neurons from microglial phagocytosis targeted inhibiting PAFR after stroke}

To reveal the impacts that neurons transferred EVs derived miR-98 to microglia after ischemic stroke, we first study the roles of miR-98 in the primary cultured microglia (Fig. 4a). Targetscan software and miRbase database were used to predict that platelet activating factor receptor (PAFR) was one of the target genes of miR-98. Transcriptional profiling analysis in a database of cell type expression categories in the cerebral cortex of mice established by Zhang $\mathrm{Y}$ et al. ${ }^{34}$ showed that PAFR was expressed almost exclusively in microglia compared with neurons in the brain. We identified that miR-98 could target and regulate PAFR through binding with the 3 '-UTR of PAFR using dual-luciferase reporter gene assay (Fig. 4b, c). Several studies indicated that PAFR is involved in the process of efferocytosis (phagocytosis of apoptotic cells) and engaged in microglial phagocytosis of synapses in the multiple sclerosis ${ }^{35,36}$. However, the role of microglial PAFR in ischemic stroke remains unclear. Therefore, we overexpressed miR-98 and downregulated PAFR, respectively (Fig. 4d-g) to identify the effects of miR-98 targeted regulating PAFR on the microglia phagocytosis.

The $\mathrm{pH}$-sensitive rhodamine-based $\mathrm{pHrodo}^{\mathrm{TM}}$ Red dyes, used as specific sensors of endocytic and phagocytic events, will undergo a marked increase in fluorescence in response to an environmental shift from extracellular high $\mathrm{pH}$ to intracellular low $\mathrm{pH}$. After $48 \mathrm{~h}$ transfection of miR-98 or PAFR, the pHrodo ${ }^{\mathrm{TM}}$ Red dyes were added into CellTrack$\mathrm{er}^{\mathrm{TM}}$ Green-labeled microglia before OGD/R treatment. The treated microglia were examined every $30 \mathrm{~min}$ by a fluorescence microscope. The time course of increased red fluorescence documented the accumulation of pHrodoconjugated zymosan bioparticles. We found microglial phagocytosis peaked at $120 \mathrm{~min}$ after OGD $3 \mathrm{~h}$ and reoxygenation. Both miR-98 overexpression and PAFR downregulation could inhibit the red bioparticles phagocytosis of microglia and the increases of red fluorescence (Fig. 4h, i). Finally, we investigated whether miR-98 overexpression could prevent neurons from microglial phagocytosis. As shown in Fig. 4j and $\mathrm{k}$, microglia were "friendly" symbiotic with surviving neurons before OGD/R treatment and microglia shifted into phagocytes (Fig. 4l) and attacked injured neurons after OGD$\mathrm{R}$ treatment. MiR-98 overexpression or PAFR downregulation could inhibit the microglial phagocytosis of surviving neurons (Fig. 4k, m). The results suggest that miR-98 targeting PAFR could prevent salvageable neurons from microglial phagocytosis after OGD/R treatment.

To further address our speculations in vivo, miR-98 agomir was injected into the lateral ventricle 3 days before C57BL/6 J mice were subjected to focal cerebral ischemia (Fig. 5a, b). Previous studies indicated that phagocytic microglia were observed soon 1 day after MCAO, peaked at 3 days after $\mathrm{MCAO}^{37}$. Blocking microglia engulfing stress but viable neurons may be beneficial after ischemic stroke $^{28,30}$. Therefore, we tested microglial phagocytosis on the first day and 3rd day after ischemic stroke. FISH was used to determine the expression of miR-98 (Fig. 5c). 


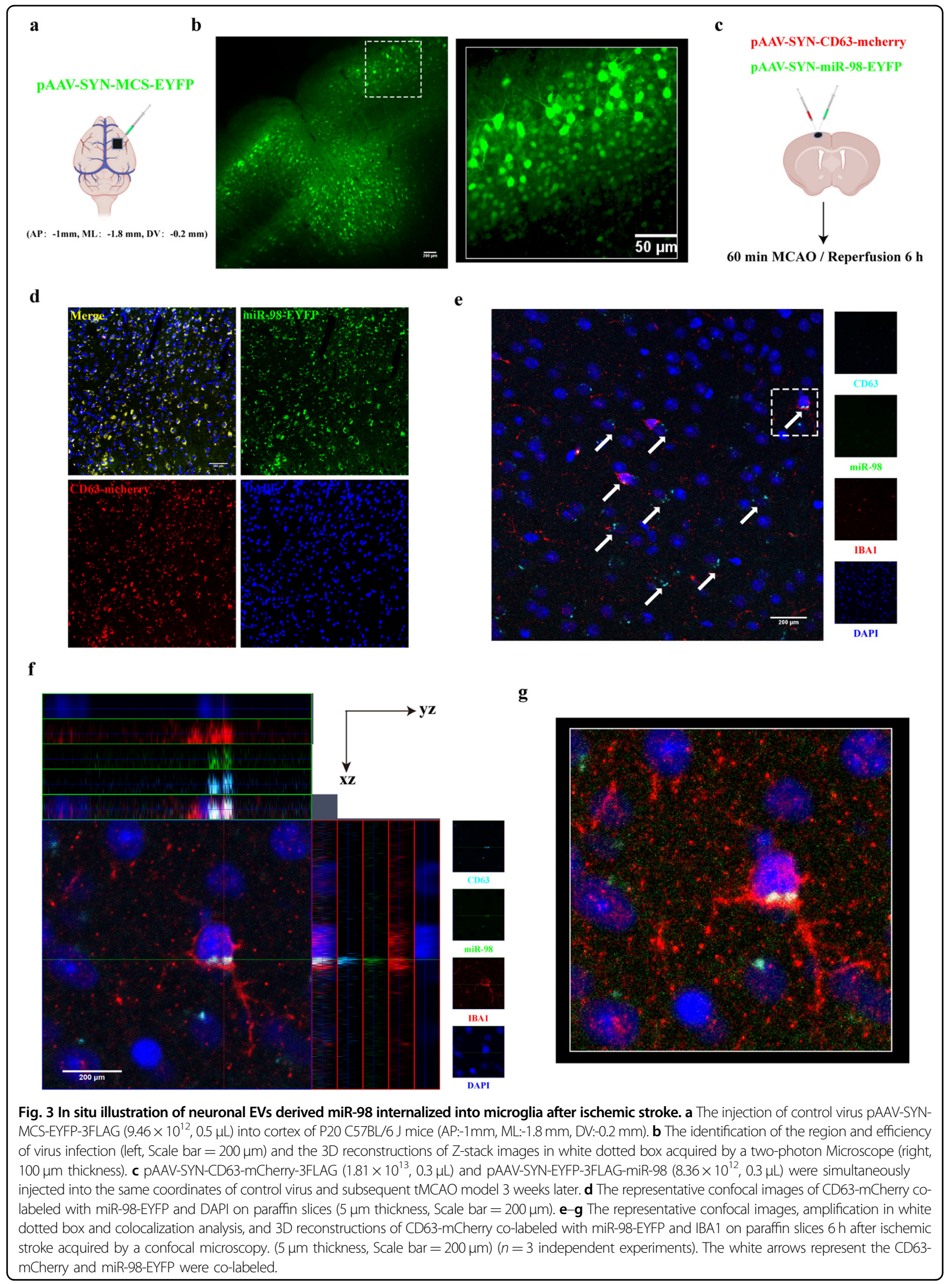




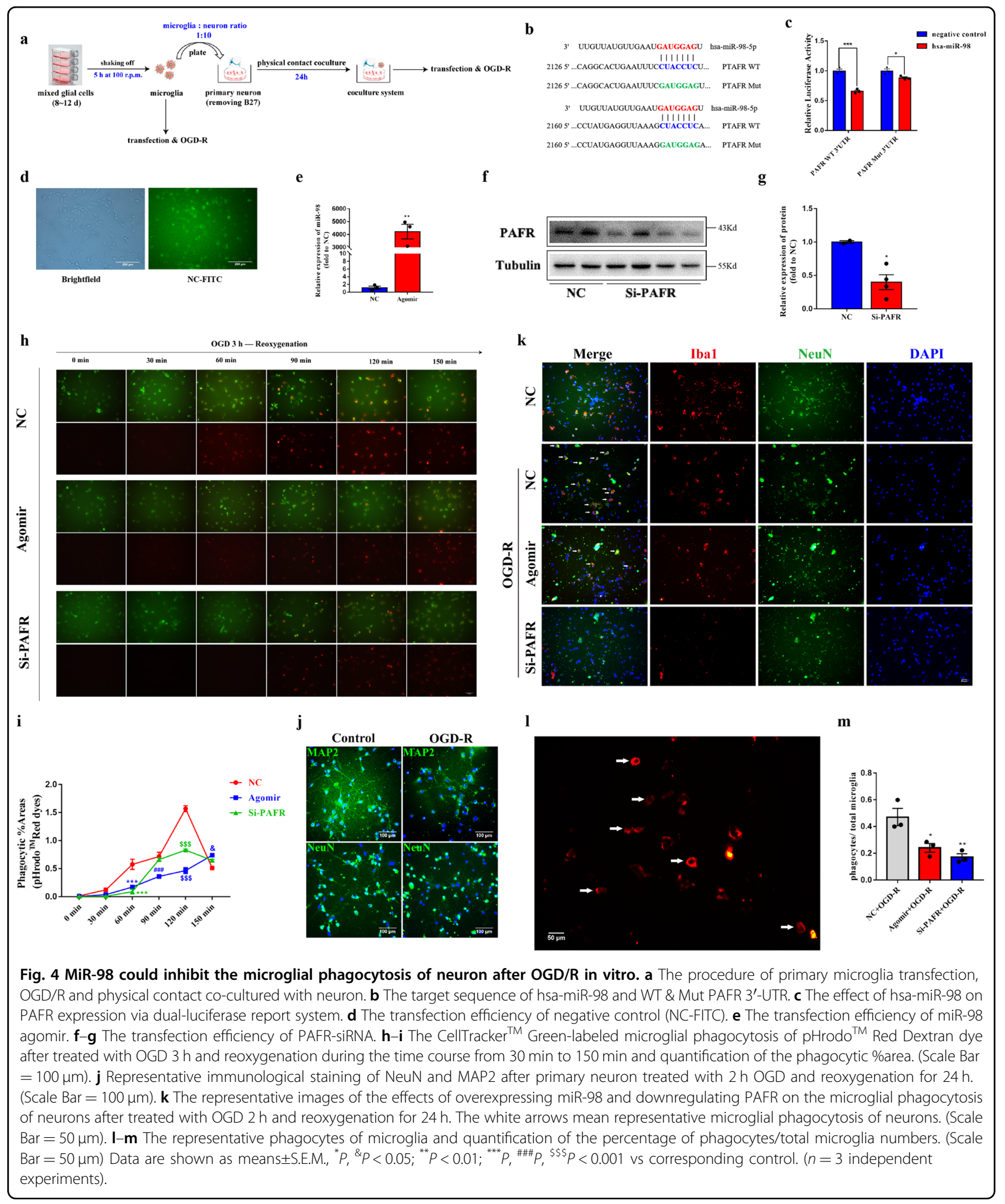

NeuN and IBA1were utilized to label neurons and microglia. The results showed that in normal conditions, microglia were branched and some were adjoined with neurons, with a rather low expression of PAFR (Fig. 5d).
On the first day after ischemic stroke, the microglia activated and could phagocytize some dead neuronal fragments. At this time, miR-98 agomir treatment did not obviously affected microglial phagocytosis in the 


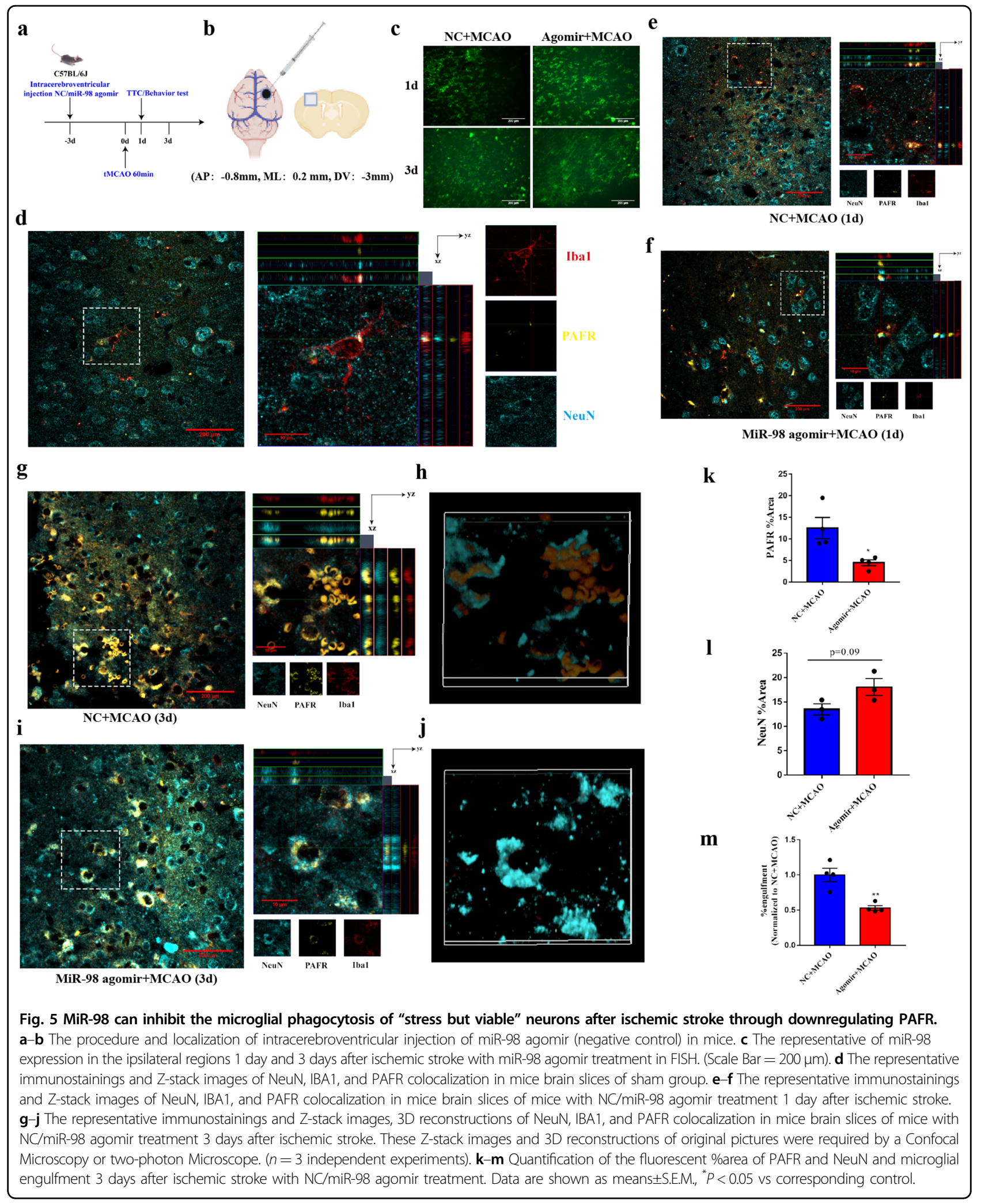

ipsilateral lesions (Fig. 5e, f). On the third day after ischemic stroke, microglia abundantly shifted into phagocytes and vigorously ate stressed but viable neurons in the lesions. Strikingly, Z-stack images and 3D reconstructions showed that miR-98 agomir treatment significantly inhibited microglial phagocytosis of dying 
neurons in the ipsilateral lesions (Fig. $5 \mathrm{~g}-\mathrm{j}$, m, Supplementary Fig. 5 \& 8, Supplementary video 1, 2). Collectively, the above results in vitro and in vivo suggest that overexpression of miR-98 blocks microglial phagocytosis of stress but salvageable neurons possibly by inhibiting PAFR (Fig. 5k) and subsequently attenuate neuronal death (Fig. 5l) in acute ischemic stroke.

\section{Overexpression of miR-98 can reduce neuronal damages in ischemic stroke in in vitro and in vivo}

Microglia activation can cause neurotoxicity via excess nitric oxide $(\mathrm{NO})$ generation by inducible NO synthase (iNOS). High levels of NO induce neuronal death by causing inhibition of mitochondrial cytochrome oxidase in neurons ${ }^{38}$. Accordingly, we detected the iNOS expression of microglia after ischemic stroke. The results showed that miR-98 agomir inhibited iNOS expression significantly on the 3rd day after ischemic stroke (Fig. 6a). Simultaneously, we verified that miR-98 agomir reduced the expressions of iNOS in microglia (Fig. 6b, c) and relieved OGD-R-induced cell injury determined by lactate dehydrogenase assay (Fig. 6d). Previous studies reported that PAFR-mediated signaling pathway regulated the iNOS production ${ }^{39}$, our results also demonstrated that miR-98 agomir downregulated PAFR and reduced the expression of iNOS (Fig. 6e, f). In addition, we observed the effects of miR-98 in the LPS-induced inflammatory model of microglia. We found overexpression of miR-98 inhibited LPS-induced microglial activation (Fig. 6g). Overexpression of miR-98 could downregulate PAFR and thereby reduce the expression of iNOS (Fig. 6h-k), whereas could not reverse the expression of CD206 which is thought as an anti-inflammatory marker (Supplementary Fig. 6). Altogether, these results indicated that miR98 can reduce iNOS production by inhibiting PAFR and attenuate inflammatory damage.

We further demonstrated that miR-98 could alleviate neural injury both in mice and rats after ischemic stroke. The results showed that miR-98 agomir could significantly increase the Nissl positive cells, suggesting relieving the neuronal damages induced by ischemia both in cortex and striatum (Fig. 6l, m). Subsequently, miR-98 agomir reduced the infarctions and attenuated the neurological deficits both in mice and rats (Fig. $6 n-s$ ).

\section{Discussion}

We show herein that neurons communicate with microglia via EVs to remodel the brain after ischemic stroke. Our results provide evidences that EVs derived miR-98 can act as an intercellular signal to regulate microglia engulfing neurons after ischemic stroke (Fig. 6t). Our data support the concept that non-cell autonomous signaling is vital for CNS recovery after injury or disease.
Although the pathogenesis of ischemic stroke has been investigated for decades, preventing delayed neuronal death is still the therapeutic direction. However, there are currently no precise blood-based biomarkers with clinical utility for acute ischemic stroke. Studies reported that miRNAs showed promise for ischemic stroke owing to their cell type-specific expression patterns and stability in peripheral blood ${ }^{40,41}$. Therefore, we attempted to explore their utility as an early diagnostic marker and potential therapeutic value for ischemic stroke. In our study, we first found miR-98 was decreased obviously in the serum of acute ischemic stroke patients. The similar decrease of miR-98 expression was also observed in the serum of ischemic stroke rats induce by tMCAO. Furthermore, we demonstrated that the serum levels of EVs miR-98 were decreased in both individuals and rats likewise, which indicate that miR-98 might be a potential biomarker for ischemic stroke.

MiR-98, as a member of let-7 group, has been reported to participated in several neurological diseases such as Alzheimer's Disease ${ }^{42}$, neuroblastoma ${ }^{43}$ and neuroinflammatory conditions ${ }^{17}$. Whereas, the specific effects and mechanisms of miR-98 involved in the pathophysiological progress of ischemic stroke remain unknown. To determine the critical periods and function of miR-98 participating in the ischemic stroke, we utilized rats to establish ischemic stroke model and observed the changes of miR-98 in the penumbra field of rats 1 day to five weeks after ischemia. Of note, we observed that the expression level of miR-98 kept from decrease on the 1st day but dropped significantly on the $3 \mathrm{rd}$ day post-ischemia. Simultaneously, we found miR-98 expression peaked at $3 \mathrm{~h}$ and dropped markedly at $5 \mathrm{~h}$ after OGD/R. These data suggest that miR-98 could serve as an endogenous protective factor for the acute phase of ischemic stroke. Previous studies mentioned that ischemic neurons could not only activate self-preservation but also release extracellular "help me" signals to adjacent cells to limit damage. Based on this, we guessed that miR-98 could function as a signal mediating communication between neurons and other neural cells after ischemic stroke. EVs are a novel form of information exchange among neural cells within the nervous system, via carrying and transferring cargo miR$\mathrm{NAs}^{44-47}$, and regulate brain remodeling process after ischemic stroke ${ }^{12,48}$. Our results showed that the expression alteration of miR-98 was consistent to the time of microglial phagocytosis change ${ }^{37}$, suggesting that miR-98 might be involved in the neurons and microglia interaction. Microglia are brain-resident immune cells that mediate key functions to support the CNS. Microglia express a wide range of molecular sensors, which recognize exogenous or endogenous insults and initiate an immune response ${ }^{21,49}$. In addition, microglia also act as guardians in the brain by promoting phagocytic clearance and providing trophic 


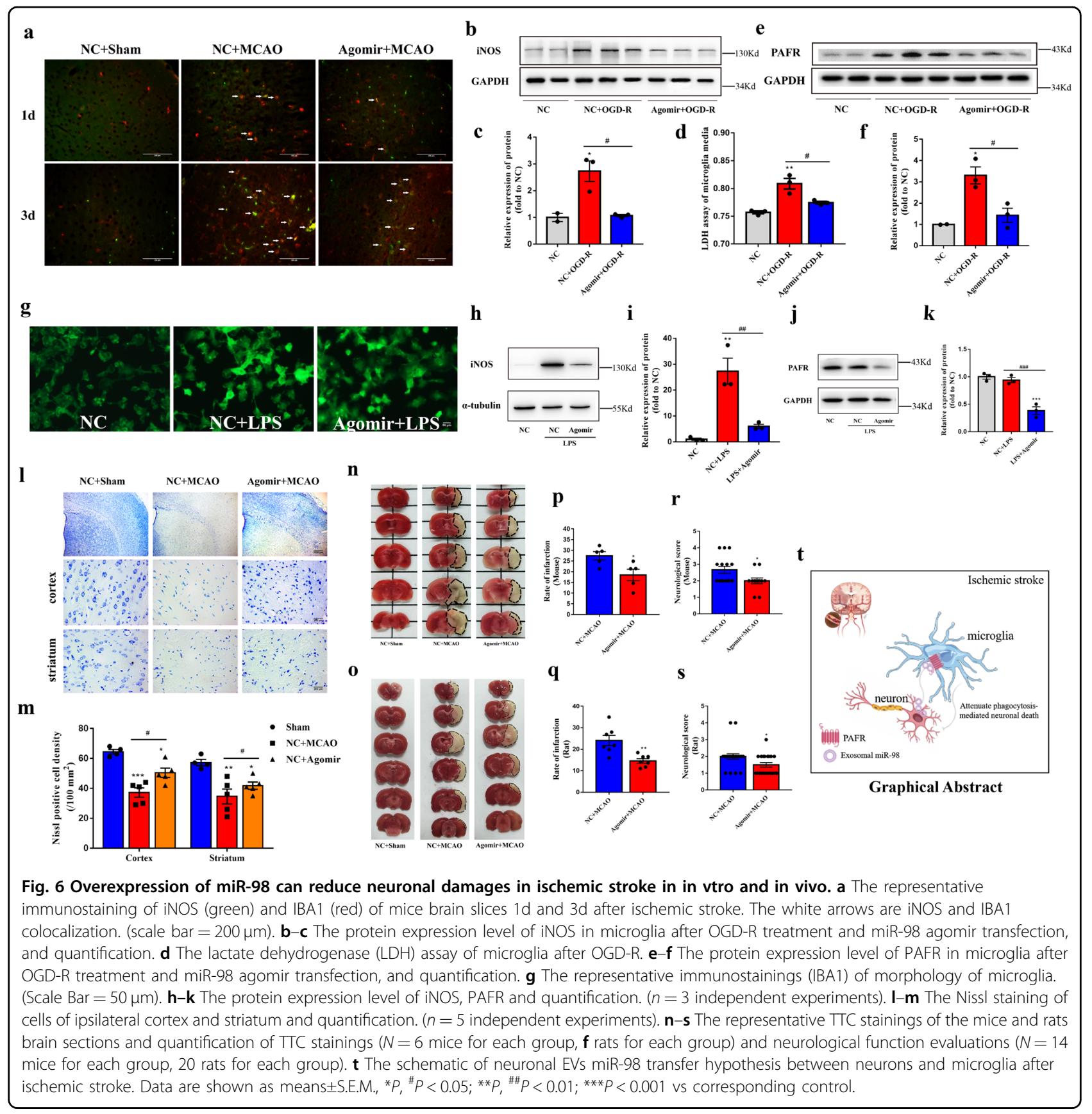

support to ensure tissue repair and maintain cerebral homeostasis $^{50}$. Microglial activation is considered as an early, sensitive and credible signal for neuronal damage in progress of ischemic stroke ${ }^{37}$. Therefore, we hypothesized that neuronal EVs derived miR-98 could act as a "help me" signal and be released to microglia.

Our present work demonstrated that neurons could transfer miR-98 via EVs secretion to prevent the stressbut-viable neurons from microglial phagocytosis after ischemic stroke. PAFR was reported to regulate phagocytosis in the neurological diseases as mentioned previously ${ }^{35,36}$. In our work, we performed experiments in vitro and in vivo to demonstrated that miR-98 could target PFAR and thereby inhibit the microglial phagocytosis, and thereby attenuate ischemia/OGD-induced neuronal death. Finally, we confirmed that miR-98 agomir given ahead could alleviate neurological deficit and infarction of ischemic stroke in rats and mice, which further confirmed that miR-98 exerted endogenous protective effects. Therefore, our data show that miR-98 regulates microglial phagocytosis through targeting PFAR, and thereby plays neuroprotection in stroke. 
Taken together, we reveal that EVs derived miR-98 acts as an intercellular signal mediating neurons and microglia communication during the brain remodeling after ischemic stroke. The present work provides a novel insight into the roles of neuronal EVs in the stroke pathogenesis and a new EVs derived miRNAs-based therapeutic strategy for stroke. Moreover, the serum level of miR-98 might be a potential indicator for stroke diagnosis and prognosis.

\section{Materials and methods \\ Animals}

All experiments and protocols were approved by Institutional Animal Care and Use Committee (IACUC) of Nanjing Medical University and in accordance to the rules of the Experimental Animal Application Criteria. The approval no. IACUC-1912041. Sprague-Dawley (SD) male rats weighing $250 \pm 10 \mathrm{~g}$ and 6-8-week-old C57BL/6 J male mice weighing $25 \pm 2 \mathrm{~g}$ were purchased from the Animal Resource Center of the Faculty of Medicine of Nanjing Medical University for use in the experiment. All the animals were kept in a standardized environment (temperature $25 \pm 2{ }^{\circ} \mathrm{C}$, humidity $55 \pm$ $10 \%$ ), maintained on a $12 \mathrm{~h} \mathrm{light/dark} \mathrm{cycle} \mathrm{(lights} \mathrm{on}$ from 20:00 to 08:00) and housed with access to food and water ad libitum. All the experimental animals were allowed to adapt to the new environment for 1 week and fasted for $12 \mathrm{~h}$ before the experiments. All the animals were allocated to experimental groups and processed with no randomization and the investigator was blinded to the group allocation.

\section{Human samples}

The serum samples of acute stroke patients were provided by Department of Neurology and age-matched healthy control provided by health examine center, the Second Affiliated Hospital of Nanjing Medical University. The information of human subjects is provided in Supplementary Table S1. All the subjects were informed consent for the sample collection. The study was approved by the Medical Ethical Committee of Nanjing Medical University (no. 2017-032). Equal volumes of serum from stroke patients and healthy control $(200 \mu \mathrm{L})$ were loaded for realtime quantitative reverse transcription-polymerase chain reaction (qRT-PCR) detections.

\section{Quantitative reverse transcription-polymerase chain reaction}

Real-time qRT-PCR was carried out using the SYBR Green mixture (TaKaRa, Japan) in a QuantStudio 5 system (Thermo Fisher Scientific, USA). The Total RNA was extracted from serum, tissues and cells by using a TRIzol reagent (Life Techonologies, 15596018) and prepared for quantitative reverse transcriptase PCR by using
MasterMix (TaKaRa, Japan). Reverse transcription was performed using the All-in-One ${ }^{\mathrm{TM}}$ miRNA First-Strand cDNA Synthesis Kit (GeneCopoeia, Cat. No: QP013). Total RNA (800 ng) was reverse-transcribed with $4 \mu \mathrm{L} 5 \times$ PAP/RT buffer, $0.8 \mu \mathrm{L}$ RTase Mix, $0.8 \mu \mathrm{L} 2.5 \mathrm{U} / \mu \mathrm{L}$ Poly A Polymerase and RNasefree water added to $20 \mu \mathrm{L}$. A reverse transcription reaction was performed in a thermal cycler as follows: $37^{\circ} \mathrm{C}$ for $60 \mathrm{~min}, 85^{\circ} \mathrm{C}$ for $5 \mathrm{~min}$, and held at $4{ }^{\circ} \mathrm{C}$ forever. The cycling conditions were as follows: denaturation at $95^{\circ} \mathrm{C}$ for $30 \mathrm{~s}$, followed by 40 cycles of DNA synthesis at $95^{\circ} \mathrm{C}$ for $5 \mathrm{~s}$ and $60^{\circ} \mathrm{C}$ for $34 \mathrm{~s}$. U68 was used as an endogenous control, and the relative expression of target genes was determined using the $2^{-\Delta \Delta c t}$ method. The All-in-One ${ }^{\mathrm{TM}}$ miRNA Universal Adaptor PCR Primer (GeneCopoeia, Cat NO: P01011A) was used as the reverse primer sequence of $\mathrm{U} 68$ and miR98. The forward primer sequence of rno/hsa-U68 used the All-in-One ${ }^{\mathrm{TM}}$ miRNA qPCR Primer (GeneCopoeia, Cat NO: RmiRQP9013; HmiRQP9071) and the forward primer sequence of rno/hsa-miR-98 used the All-in-One ${ }^{\mathrm{TM}}$ miRNA qPCR Primer (GeneCopoeia, Cat NO: RmiRQP0853; HmiRQP9021). The serum EVs-miRNAs detections were normalized to spiked cel-miR-39 as described previously ${ }^{51}$.

\section{Focal cerebral ischemia/reperfusion model}

$\mathrm{SD}$ male rats and C57BL/6 J male mice were both used for focal cerebral ischemia/reperfusion experiments. We used a tMCAO model by means of an intraluminal suture. In brief, animals were anesthetized with isoflurane and the right external carotid artery was exposed and a nylon monofilament coated with silicon resin (Doccol) was introduced through a small incision and advanced to the carotid bifurcation along the internal carotid artery into the middle cerebral artery. The monofilament was withdrawn to restore blood flow to the middle cerebral artery territory $1 \mathrm{~h}$ (mice) $/ 1.5 \mathrm{~h}$ (rats) after the occlusion. Focal cerebral ischemia was assessed by Laser Doppler flowmetry ${ }^{52}$. Neurological function was evaluated using a four-point scale neurological score method ( 0 , no observable deficit; 1 , forelimb flexion; 2 , decreased resistance to lateral push and immediate circling; 3 , severe rotation progressing into loss of walking or righting reflex; 4, not walking spontaneously and some degree of consciousness $)^{53}$. Animals dead in the model were excluded. The identification of ischemic core zones and penumbra field are performed as previously described ${ }^{54}$.

\section{Fluorescence in situ hybridization}

Animals were anesthetized with isoflurane and perfused with PBS followed by $4 \%$ paraformaldehyde (PFA). After post fixation with $4 \%$ PFA overnight, the brains were dehydrated using a graded series of alcohol, cleared in xylene, and embedded in paraffin. Then, coronal slices 
$(5 \mu \mathrm{m})$ were cut by a rotary microtome (LEICA, RM2245, Heidelberg, Germany). The following steps followed the BersinBio $^{\mathrm{TM}}$ Fluorescence in Situ Hybridization Kit Instruction Manual. In brief, Xylene processing sample for 15 minutes at room temperature (every 5 minutes change the xylene). Incubate twice with $100 \%$ ethanol for 5 minutes. Then dry the sample. Then add proteinase $\mathrm{K}$ mixture $(0.2 \mu \mathrm{L}$ proteinase $\mathrm{K}, 20 \mu \mathrm{L}$ Tris-Hcl, $\mathrm{pH}$ 7.6, $80 \mu \mathrm{L}$ RNasefree water) on sample and incubate $10-15$ minutes at $37^{\circ} \mathrm{C}$. Rinse slides twice with PBS for 5 minutes at room temperature. Incubate sample for 5-8 minutes with preheating denaturing solution $(70 \%$ formamide in $2 \times$ SSC $[17.6 \mathrm{gNacl}, 8.8 \mathrm{~g}$ sodium citrate in $1 \mathrm{~L}$ sterilized water]) at $78^{\circ} \mathrm{C}$. Incubate with $70 \%$ Ethanol for $5 \mathrm{~min}, 85 \%$ Ethanol for 5 minutes, and 100\% Ethanol for 5 minutes at $-20^{\circ} \mathrm{C}$. Then dry the sample. Add $2 \mu \mathrm{l}$ miR-98-5p FITC Probe (BersinBio ${ }^{\text {TM }}$, QD355) to the remaining $18 \mu \mathrm{L}$ Probe Diluent $(60 \%$ deionized formamide, $500 \mu \mathrm{g} / \mathrm{mL}$ yeast tRNA [Invitrogen, 15401011], $50 \mu \mathrm{g} / \mathrm{mL}$ Heparin sodium salt [Aladdin, 9041-08-1], 0.1\% Tween-20, 20× SSC, 9.2 mM Citric acid solution, $\mathrm{pH}$ 6.0) to obtain $20 \mu \mathrm{L}$ Hybridization reaction mixture. Mix well and denatured at $78^{\circ} \mathrm{C}$ for 5 minutes. Then incubate for 5 minutes at $37^{\circ} \mathrm{C}$. Then add $10-15 \mu l$ Hybridization reaction mixture on sample. Add lid and incubate for $16 \mathrm{~h}$ to $20 \mathrm{~h}$ at $37^{\circ} \mathrm{C}$. Rinse slides twice with preheating washing buffer for 5 minutes at $53^{\circ} \mathrm{C}$. Rinse slides twice with preheating $2 \times \mathrm{SSC}$ for 5 minutes at $37^{\circ} \mathrm{C} ; 0.5 \times \mathrm{SSC}$ for 15 minutes at $37^{\circ} \mathrm{C} ; 0.2 \times \mathrm{SSC}$ for 15 minutes at $37^{\circ} \mathrm{C}$; Rinse slides with PBS for 5 minutes at room temperature. Samples can be analyzed in a drop of fluorescence decay mounting medium (Beyotime Biotechnology, P0126) under a fluorescence microscope.

\section{Primary neuron cultures}

Primary neuron cultures were prepared from cerebral cortices of embryonic day (E) 16 18 Sprague-Dawley rat embryos $^{44}$. In brief, embryos were removed from maternal rat anesthetized with isoflurane and euthanized by decapitation. Cerebral cortices were dissected and digested with 0.125\% trypsin (Life Technologies, 27250-018) and 0.025\% DNase I (Biofroxx, 1121MG010) at $37^{\circ} \mathrm{C}$ for $15 \mathrm{~min}$. Dissociated cells were filtered with $80-\mu \mathrm{m}$ cell strainer, collected by centrifugation ( $1000 \mathrm{rpm}$ for $5 \mathrm{~min}$ ) and plated on dishes (Corning, 430167) or 24-well plates (Corning, 3524) coated with $0.01 \mathrm{mg} / \mathrm{mL}$ Poly-D-lysine (Sigma, P6407) and cultured in Dulbecco's Modified Eagle Medium (DMEM, Life Technologies, 11965-084) containing 10\% fetal bovine serum (FBS, Life Technologies, Australia, 10099-141), 50 U/ $\mathrm{mL}$ penicillin-streptomycin (Life Technologies, 15140122). At $6 \mathrm{~h}$ after seeding, the medium was changed to Neurobasal medium (Life Technologies, 21103-049) supplemented with 2\% B27 (Life Technologies, 17504044) and $50 \mathrm{U} / \mathrm{mL}$ penicillin-streptomycin. Neurons were maintained at $37^{\circ} \mathrm{C}$ in a humidified incubator of $95 \%$ air and $5 \% \mathrm{CO}_{2}$ and grown for 10 12 days with half of the media replaced every 2 days. The purity of neuron cultures was determined by immunocytochemical staining using an antibody against microtubule-associated protein-2 (MAP2), $>90 \%$.

\section{Primary microglia cultures and transfection}

Primary microglia cultures were isolated from 1-day-old postnatal SD rats. In brief, primary cultures of glial cells were obtained from the cerebral cortices, which were earlier digested by $0.125 \%$ trypsin at $37^{\circ} \mathrm{C}$ for $15 \mathrm{~min}$ and seeded into poly-D-lysine-coated $25-\mathrm{cm}^{2}$ culture flasks (Corning, 430639). The cultures were maintained for 10 days in DMEM supplemented with 10\% FBS and $50 \mathrm{U} /$ $\mathrm{mL}$ penicillin-streptomycin at $37^{\circ} \mathrm{C}$ in a humidified incubator with $5 \% \mathrm{CO}_{2}$ air atmosphere. Media were replaced every 2 3 days and grown for 9 10 days. Microglia were separated from the mixed primary culture by shaking off for $5 \mathrm{~h}$ at $100 \mathrm{rpm}$ and then planked in plates with DMEM medium containing 10\% FBS and $50 \mathrm{U} / \mathrm{mL}$ penicillin-streptomycin. Before the experiments, the percentage of the primary microglia was confirmed by Ionized calcium binding adapter molecule 1 (Iba1, WAKO, 019-19741) staining with over 97\% purity.

Primary microglia were transfected in a 6/24-well plate with miRNA agomir, miRNA antagomir and siRNAs purchased from GenePharma (Shanghai, China) using siRNA-Mate (GenePharma, G04002), according to the manufacturer's instructions. The sequences are as follows.

Rno/mmu-miR-98 agomir:

Forward: $5^{\prime}$-UGAGGUAGUAAGUUGUAUUGUU- ${ }^{\prime}$

Reverse: 5'-CAAUACAACUUACUACCUCAUU- $3^{\prime}$

Rno/mmu-miR-98 antagomir:

5'-AACAAUACAACUUACUACCUCA- $3^{\prime}$

Rno-Ptafr siRNA:

Forward: 5'-CCAACUUCCAUCAGGCUAUTT-3'

Reverse: $5^{\prime}$-AUAGCCUGAUGGAAGUUGGTT- $3^{\prime}$

Negative control-FITC:

Forward: 5'-UUCUCCGAACGUGUCACGUTT-3'

Reverse: 5'-ACGUGACACGUUCGGAGAATT-3'.

\section{Oxygen and glucose deprivation and reoxygenation (OGD-R)}

OGD experiments were performed using an incubator (Thermo Fisher Scientific, Waltham, USA) with premixed gas $\left(95 \% \mathrm{~N}_{2}\right.$ and $\left.5 \% \mathrm{CO}_{2}\right)$ kept at $37{ }^{\circ} \mathrm{C}$. To initiate OGD, cells culture medium was replaced with deoxygenated, glucose-free DMEM (Life Technologies, 11966-025). After OGD, the cells were then transferred to a normoxic incubator $\left(95 \%\right.$ air and $5 \% \mathrm{CO}_{2}$ ) replaced with normal medium. Although the time of OGD challenge and reoxygenation could be adapted according to different cells and culture system. 


\section{NCM preparation}

NCM was harvested from neurons grown for 10 12 days replaced with Neurobasal medium without B27 supplement (starvation control) or OGD $30 \mathrm{~min}$ and reoxygenation $3 \mathrm{~h}$ plus GW4849 (EV secretion blocker, $20 \mu$ M, Santa Cruz Biotechnology, sc-218578). NCM from neurons was cleared by centrifugation ( $1000 \mathrm{rpm}$ for $5 \mathrm{~min}$ ) and then diluted to 3:1 by mixing with microglial complete medium to treat microglia. NCM was collected simultaneously and used in parallel without storage. After treated $24 \mathrm{~h}$, microglia were collected for the next detections.

\section{Primary neuron-microglia physical coculture}

To generate neuron-microglia physical coculture ${ }^{55}$, cortical neurons $\left(1 \times 10^{6}\right.$ cells $\left./ \mathrm{mL}\right)$ were cultured in 24 well plates and grown for 8 9 days replaced with neurobasal medium without B27 supplement, which inhibits the growth of glial cells. Primary microglia $\left(1 \times 10^{5}\right.$ cells $\left./ \mathrm{mL}\right)$ were seeded and cultures 1:10 with neurons. After $24 \mathrm{~h}$, the coculture system was subjected to OGD/R treatment.

\section{Isolation of serum and neuronal EVs}

Neuronal EVs were isolated from the harvested conditioned medium of rat cortical neurons cultured 10 12 days in vitro. Every $5-6 \times 10^{6}$ neurons were cultured in a 100 $\mathrm{mm}$ dish and $10 \mathrm{~mL} \mathrm{NCM}$ were collected. The EVs were harvested from 10 dishes NCM supernatant $(100 \mathrm{~mL})$ and isolated by multi-step centrifugation as previously reported with minor modification. Serum EVs were isolated as the same. Briefly, the supernatant was centrifuged at $300 \times g$ for $10 \mathrm{~min}, 2000 \times g$ for $10 \mathrm{~min}$, and $16,500 \times g$ for $30 \mathrm{~min}$ at $4{ }^{\circ} \mathrm{C}$ to remove cells and debris and then filtered using a $0.22-\mu \mathrm{m}$ filter (Millipore, SLGV033RB). The filtrate was centrifuged at $140,000 \times g$ for $2 \mathrm{~h}$ at $4{ }^{\circ} \mathrm{C}$ in a Type Ti70 rotor using an L-100XP ultracentrifuge (Beckman Coulter, Brea, CA, USA). The EV pellet was resuspended in PBS and ultracentrifuged again at $140,000 \times g$ for $2 \mathrm{~h}$. The isolated pellets were suspended in sterile $1 \times \mathrm{PBS}$ for EV characterization and culture experiments ${ }^{56}$.

\section{Identification of EVs by TEM, nanoparticle tracking analysis} (NTA), and western blotting

The EVs were diluted in sterile PBS, fixed with $1 \%$ glutaraldehyde, applied onto a carbon-coated copper grid, and stained with $1 \%$ phosphotungstic acid. A TEM (Tecnai Spirit Biotwin) was employed to observe the specimens. The average particle size and zeta potential of neuronal and serum EVs were investigated with a Zetasizer (Nano-ZS90, Malvern, England) ${ }^{57}$. Western blotting was applied to detect EV markers carried out with antiAlix (PDCD61P, Proteintech, Cat\# 12422-1-AP, 1:1000), anti-TSG101 (Proteintech, Cat\# 14497-1-AP, 1:1000) antibodies and negative marker, anti-Calnexin (Proteintech, Cat\# 10427-2-AP, 1:1000) antibody.

\section{Labeling of neuronal EVs and uptake by microglia}

To detect the uptake of neuronal EVs by microglia in vitro, neuronal EVs were labeled by a PKH26 Red Fluorescent Cell Linker Kit (Sigma-Aldrich, MINI26$1 \mathrm{KT}$ ). In brief, $1.5 \mu \mathrm{L}$ PKH26 was diluted in $100 \mu \mathrm{L}$ diluent $\mathrm{C}$, and $80 \mu \mathrm{L}$ diluent $\mathrm{C}$ was added to the EVs resuspended in $20 \mu \mathrm{L}$ PBS. Subsequently, transfer the EVs mixed with diluent $\mathrm{C}$ to the tube containing PKH26 in diluent $\mathrm{C}$. After incubation for $5 \mathrm{~min}$, the reaction was stopped by adding $100 \mu \mathrm{L}$ PBS containing 10\% EVs-free FBS (FBS was centrifuged at $140,000 \times g$ for $2 \mathrm{~h}$ using a Ti70 rotor to deplete $\mathrm{EVs})^{58}$. Then, the EVs were centrifuged at $140,000 \times g$ for $2 \mathrm{~h}$ using an SW55Ti rotor to remove uncombined dye. After washing with PBS, the labeled EVs were resuspended. The labeled neuronal EVs were then incubated with CellTracker ${ }^{\mathrm{TM}}$ Green CMFDA Dye (Invitrogen, C2925)-labeled microglia for $12 \mathrm{~h}$. The addition of EVs isolated from $100 \mathrm{ml}$ neuronal-conditioned media into 24-well-plate microglia culture medium was counted as concentrating 200 times (200x).

\section{The detection of microglial phagocytosis}

Primary microglia were seeded in 24-well-plates incubated overnight in culture medium, and processed according to the pHrodo $^{\mathrm{TM}}$ Red zymosan bioparticles conjugate for phagocytosis (Life Technologies, P35364) procedure. Microglia were labeled with CellTracker ${ }^{\mathrm{TM}}$ Green $(1 \mu \mathrm{M}$ Dye was added into PBS and mixed for $30 \mathrm{~min})$. After twice washed with PBS, microglia were incubated with PBS containing $10 \mu \mathrm{L} / \mathrm{ml}$ of pHrodo Red zymosan bioparticles at $37^{\circ} \mathrm{C}$ for $15 \mathrm{~min}$ (if microglia were treated with OGD, incubated when reoxygenation). The treated microglia were examined every $30 \mathrm{~min}$ by a fluorescence microscope (Zeiss, Heidelberg, Germany).

\section{In vitro luciferase assays}

The 3'-UTRs of ptafr mRNA harboring the predicted miR98 binding sequences were PCR amplified from human genomic DNA and cloned into Bam HI and FXho I of the pLUC-Report luciferase vector (Shenzhen Kangbio Biological Technology Co., Ltd, Shenzhen, Guangdong) to generate the ptafr-3'-UTR reporter construct. Mutagenesis of predicted targets with a mutation of $7 \mathrm{bp}$ from the site of perfect complementarity was performed using a site-directed Mutagenesis Kit (Takara, 638909) ${ }^{59}$. HEK $293 \mathrm{~T}$ cells were plated at a density of $2 \times 10^{4}$ cells/well in 96-well plates before transfection. When cells were grown to $50 \%$ confluence in 96-well plates, they were co-transfected with $0.2 \mu \mathrm{g}$ plasmid DNA, $0.15 \mu \mathrm{g}$ sensor reporter gene, and $0.45 \mu \mathrm{g}$ miR-98 mimics or miRNA negative control (NC) using Lipofectamine 2000 (Invitrogen, 11668027) according to the manufacturer's instructions. Luciferase activity was detected with a Dual-Luciferase Reporter Assay System (Promega, Cat \#E1980) $48 \mathrm{~h}$ after transfection (using miR-101 targeting 
EZH2 as positive control, supplementary Fig. 3). Firefly luciferase activity was normalized to Renilla luciferase activity for each transfected well.

The primers of Ptafr-miR-98 FXho I:

5'-cacaactcgagTCATTTCCTGTGTACCGGGC-3'

The primers of Ptafr-miR-98R Bam HI:

5'-aaggatccTAAGGGACCTGCAAAGCCTG-3'

The primers of Ptafr-miR-98-MR:

5'-CTCCATCCTTTAACCTCATAGGTAATGACCCT

AACTCCATCGAAATTCAGTGCCTGGT-3'

The primers of Ptafr-miR-98-MF:

5'-GATGGAGTTAGGGTCATTACCTATGAGGTTAA AGGATGGAGATGGGATTGTTATACGCC-3'.

\section{Drug treatment}

Microglia were treated with LPS $(0.01 \mu \mathrm{g} / \mathrm{mL}$; SigmaAldrich, L2880) to establish inflammatory model in vitro. MiR-98 agomir was injected intracerebroventricularly (i.c.v) in vivo transfection technology. Entranster ${ }^{\mathrm{TM}}$-in vivo transfection reagent (18668-11-1) was purchased from Engreen (Beijing, China) for in vivo, which is low toxic, efficient and no obvious inflammatory injury to animals. The in vivo transfection was performed according to the specific instructions. In brief, the negative control and rno/ mmu-miR-98 agomir $(1 \mu \mathrm{g} / 1 \mu \mathrm{L})$ were added to $0.5 \mu \mathrm{L}$ of Entranster $^{\mathrm{TM}}$ in vivo transfection reagent. The solution was mixed gently, left for $15 \mathrm{~min}$ and then injected.

\section{Intracerebroventricular injection}

For the injection of rno/mmu-miR-98 agomir, SD rats or $\mathrm{C} 57 \mathrm{BL} / 6 \mathrm{~J}$ mice were anesthetized with isoflurane and received intracerebroventricular injection using a micro syringe under the guidance of stereotaxic instruments (RWD Life Science) 3 days before tMCAO model ${ }^{60}$. The stereotaxic coordinates of rats (AP: $-0.8 \mathrm{~mm}$, ML: $1.5 \mathrm{~mm}, \mathrm{DV}:-4.0 \mathrm{~mm}$ ) and mice (AP: $-0.8 \mathrm{~mm}$, ML: $0.2 \mathrm{~mm}, \mathrm{DV}:-3 \mathrm{~mm})$.

\section{Viral injection}

pAAV-SYN-MCS-EYFP-3FLAG $\left(9.46 \times 10^{12}, \quad 0.5 \mu \mathrm{L}\right)$, pAAV-SYN-CD63-mCherry-3FLAG $\left(1.81 \times 10^{13}, 0.3 \mu \mathrm{L}\right)$, and pAAV-SYN-EYFP-3FLAG-miR-98 $\left(8.36 \times 10^{12}, 0.3 \mu \mathrm{L}\right)$ purchased from Obio Technology (Shanghai, China) were unilaterally injected into cortex of P20 C57BL/6 J mice (AP: $-1 \mathrm{~mm}, \mathrm{ML}:-1.8 \mathrm{~mm}$, DV: $-0.2 \mathrm{~mm}$ ). Viral titers over $1 \times 10^{12}$ genomic particles $/ \mathrm{mL}$ were used and injected into each location at $100 \mathrm{~nL} / \mathrm{min}$. The following experiments were performed 3 weeks after virus injection. The 24h-post-fixed brain injected with pAAV-SYN-MCS-EYFP3FLAG was stored in $4{ }^{\circ} \mathrm{C}$ PBS and horizontally sectioned at $100 \mu \mathrm{m}$ slices using a VT1200S Vibratome (Leica). Images were visualized and captured by a confocal microscopy (Nikon A1RSi, Tokyo, Japan) to confirm the regional expression of virus infection.

\section{Triphenyltetrazolium chloride staining}

The infarct volume was evaluated $24 \mathrm{~h}$ after tMCAO. Animals were anesthetized with isoflurane, euthanized and the brains were removed. Each brain was coronally sectioned into 1 or $2 \mathrm{~mm}$ slices. The slices were incubated with $2 \%$ triphenyltetrazolium chloride (Sigma-Aldrich, T8877) at $37^{\circ} \mathrm{C}$ for $20 \mathrm{~min}$ to determine the size and extent of the infarction. The pallor area indicated ischemic infarct. The brain infarct areas were analyzed with Image-Pro Plus 6.0 to estimate the infarct volume.

\section{Nissl staining}

The paraffinized brain samples were treated with Nissl Staining Solution (Beyotime Biotechnology, C0117). A bluish-purple color was observed to display the basic nervous structure of the brain. Large and numerous Nissl bodies indicated that the nerve cells had a high ability to synthesize proteins. The number of Nissl corpuscles decreased significantly when the nerve cells were damaged. Images were captured by a fluorescence microscope (Zeiss, Heidelberg, Germany). The number of stained cells was counted from the selected fields randomly and analyzed with Image-Pro Plus 6.0.

\section{Western blotting}

Cell samples were dissociated in $100 \mu \mathrm{L}$ of lysis buffer consisting of $1 \%$ phenylmethanesulfonyl fluoride (KeyGEN, KGP610). The protein concentrations were determined with a BCA Protein Assay kit (Beyotime Biotechnology, P0012), and the proteins were denaturalized with $5 \times$ loading buffer. Total proteins $(40 \mu \mathrm{g})$ were separated by sodium dodecyl sulfate-polyacrylamide gel electrophoresis and transferred to polyvinylidene fluoride membranes (Roche, 3010040001). Then, 5\% skim milk in Tris-buffered saline-Tween-20 (TBST) $(1 \mathrm{M}$ Tris- $\mathrm{HCl}$ ( $\mathrm{pH} 7.5), 0.8 \% \mathrm{NaCl}$, and $0.1 \%$ Tween-20) was used to block the membranes for $1 \mathrm{~h}$ at room temperature. The membranes were then incubated with the following primary antibodies at $4{ }^{\circ} \mathrm{C}$ overnight: Ptafr (abcam, Cat\# ab104162, 1:200), iNOS (Santa Cruz Biotechnology, sc7271, 1:1000), CD206 (abcam, Cat\# ab64693, 1:1000), GAPDH (Proteintech, Cat\# 10494-1-AP, 1:5000), $\alpha$-tubulin (Proteintech, Cat\# 10094-1-AP, 1:2000).

\section{Immunofluorescence and confocal image acquisition}

Mice were anesthetized with isofluorane and perfused with PBS followed by $4 \%$ PFA. After post fixation with $4 \%$ PFA overnight, the brains were dehydrated using a graded series of alcohol, cleared in xylene, and embedded in paraffin. Then, coronal slices $(5 \mu \mathrm{m})$ were cut by a rotary microtome (LEICA, RM2245, Heidelberg, Germany). After deparaffinization, sections of the brain were incubated in $3 \% \mathrm{H} 2 \mathrm{O} 2$ for $15 \mathrm{~min}$ to block endogenous peroxidases for antigen retrieval and then washed with PBS. Next, sections were 
incubated with $5 \%$ goat serum and $0.01 \%$ Triton- 100 (dissolved in PBS) for $1 \mathrm{~h}$ and then stained with primary antibodies (IBA1, goat, abcam, Cat\# ab5076, 1:500; IBA1, rabbit, WAKO, 019-19741, 1:500; NeuN, Millipore, MAB377, 1:400; Ptafr, abcam, Cat\# ab104162, 1:200; iNOS, Santa Cruz Biotechnology, sc-7271, 1:500) overnight at $4{ }^{\circ} \mathrm{C}$. Following washing with PBS, the sections were incubated with Alexa Fluor 488, 555, 594, and (or) 647 donkey anti-mouse, antigoat, and (or) anti-rabbit secondary antibodies (1:1000 dilution, Invitrogen Life Technologies, NY, USA). Images were captured by a confocal microscopy or two-photon Microscope (Zeiss LSM880 with NLO \& Airyscan) after incubation with 4-6-diamidino-2-phenylindole (Beyotime Biotechnology, C1005) for $15 \mathrm{~min}$ and washing with PBS. 3D representing of Z-stack images of neuronal EVs delivered to microglia in vivo and phagocytic uptake of neurons by microglia were acquired and 3D reconstructions were generated using ZEN software (blue/black edition). The microglial engulfment was analyzed using IMARIS software (Bitplane).

\section{Statistical analysis}

Data were analyzed in a blinded way. Statistical analyses were performed using SPSS software, version 18.0 (SPSS Inc., Chicago, IL, USA). Multiple comparisons were conducted using one-way analysis of variance (ANOVA) and Tukey's multiple comparisons test. Two-way ANOVA test was used when graphs are compared between brain areas or time of treatment. The means of the two treatment groups were analyzed using unpaired or paired Student's $t$ tests. Data are expressed as means \pm standard error of measurements. A value of $p<0.05$ indicates that the difference was statistically significant.

\section{Acknowledgements}

The authors acknowledge Professor Hongliang Xin (Nanjing Medical University) for technical support in nanoparticle tracking experiments and analysis. Funding: the National Natural Science Foundation of China (no. 81973301 and 81773701), Medical Research Project of Jiangsu Commission of Health (no. ZDA2020006), Jiangsu Provincial Key Research and Development Program (no. BE2017737), the Natural Science Research of Jiangsu Higher Education Institutions of China (no. 18KJA310004), the Major Project of Nanjing Medical University (no. NMUD2018008), and the Postgraduate Research and Practice Innovation Program of Jiangsu Province (no. KYCX19_1121 and KYCX20_1417).

\section{Author details \\ 'Department of Pharmacology, Neuroprotective Drug Discovery Key Laboratory, Jiangsu Key Laboratory of Neurodegeneration, Center for Global Health, Nanjing Medical University, Nanjing, China. ${ }^{2}$ Zhongda Hospital, Southeast University, Nanjing, China. ${ }^{3}$ The First Affiliated Hospital of Nanjing Medical University, Nanjing, China. ${ }^{4}$ Nanjing University of Chinese Medicine, the Affiliated Hospital of Nanjing University of Chinese Medicine, Nanjing, China}

\section{Author contributions}

Jin Yang was involved in the conception and design, performing experiments, acquisition, analysis, and interpretation of data and writing the paper; Lu-Lu Cao performed the experiments and was involved in the acquisition and interpreting the data; Xi-Peng Wang and Wei Guo performed the experiments and revised the article; Ruo-Bing Guo, Yu-Qin Sun, Teng-Fei Xue, Zhen-Yu Cai performed the experiments; Hong Cheng revised the article; Xiu-Lan Sun was involved in conception and design, acquisition, analysis, and interpretation of data; revising the article critically for important intellectual content; and final approval of the version to be published. All authors reviewed, provided substantive input, and approved the final manuscript.

\section{Conflict of interest}

The authors declare that they have no conflict of interest to report. All authors concur with the submission of this manuscript. The data have not been previously reported and are not under consideration for publication elsewhere.

\section{Ethics}

Animal experiments: all experiments and protocols were approved by the Institutional Animal Care and Use Committee (IACUC) of Nanjing Medical University and in accordance with the rules of the Experimental Animal Application Criteria. Approval No. IACUC-1912041. Human samples: all the subjects were informed consent for the sample collection. The study was approved by the Medical Ethical Committee of Nanjing Medical University (no. 2017-032).

\section{Publisher's note}

Springer Nature remains neutral with regard to jurisdictional claims in published maps and institutional affiliations.

Supplementary Information accompanies this paper at (https://doi.org/ 10.1038/s41419-020-03310-2).

Received: 15 July 2020 Revised: 1 December 2020 Accepted: 3 December 2020

Published online: 06 January 2021

\section{References}

1. Norris, G. T. et al. Neuronal integrity and complement control synaptic material clearance by microglia after CNS injury. J. Exp. Med. 215, 1789-1801 (2018).

2. Norving, B. Stroke management - recent advances and residual challenges. Nat. Rev. Neurol. 15, 69-71 (2019).

3. Quinn, T. J. \& Drozdowska, B. A. Stroke prediction and the future of prognosis research. Nat. Rev. Neurol. 92, e1517-e1525 (2019).

4. Lo, E. H., Dalkara, T. \& Moskowitz, M. A. Mechanisms, challenges and opportunities in stroke. Nat. Rev. Neurosci. 4, 399-415 (2003).

5. Xing, C. \& Lo, E. H. Help-me signaling: Non-cell autonomous mechanisms of neuroprotection and neurorecovery. Prog. Neurobiol. 152, 181-199 (2017).

6. Biber, K., Neumann, H., Inoue, K. \& Boddeke, H. W. G. M. Neuronal 'On' and 'Off' signals control microglia. Trends Neurosci. 30, 596-602 (2007).

7. Zhang, G. \& Yang, P. A novel cell-cell communication mechanism in the nervous system: exosomes. J. Neurosci. Res. 96, 45-52 (2017).

8. Zhang, H. G. \& Grizzle, W. E. Exosomes: a novel pathway of local and distant intercellular communication that facilitates the growth and metastasis of neoplastic lesions. Am. J. Pathol. 184, 28-41 (2014).

9. Zhang, L. et al. Microenvironment-induced PTEN loss by exosomal microRNA primes brain metastasis outgrowth. Nature 527, 100-104 (2015).

10. Hessvik, N. P. \& Llorente, A. Current knowledge on exosome biogenesis and release. Cell. Mol. Life Sci. 75, 193-208 (2017).

11. Vlassov, A. V., Magdaleno, S., Setterquist, R. \& Conrad, R. Exosomes: current knowledge of their composition, biological functions, and diagnostic and therapeutic potentials. Biochim. Biophys. Acta 1820, 940-948 (2012).

12. Zhang, Z. G. \& Chopp, M. Exosomes in stroke pathogenesis and therapy. J. Clin. Investig. 126, 1190-1197 (2016).

13. Yang, J., Zhang, X., Chen, X., Wang, L. \& Yang, G. Exosome mediated delivery of miR-124 promotes neurogenesis after ischemia. Mol. Ther. Nucleic Acids 7, 278-287 (2017).

14. Chen, J. \& Chopp, M. Exosome therapy for stroke. Stroke 49, 1083-1090 (2018).

15. Jarmalaviciute, A. \& Pivoriunas, A. Exosomes as a potential novel therapeutic tools against neurodegenerative diseases. Pharm. Res. 113, 816-822 (2016).

16. Goldie, B. J. et al. Activity-associated miRNA are packaged in Map1b-enriched exosomes released from depolarized neurons. Nucleic Acids Res. 42, 9195-9208 (2014). 
17. Rom, S., Dykstra, H., Zuluaga-Ramirez, V., Reichenbach, N. L. \& Persidsky, Y. miR98 and let-7g* protect the blood-brain barrier under neuroinflammatory conditions. J. Cereb. Blood Flow. Metab. 35, 1957-1965 (2015).

18. Bernstein, D. L. et al. miR-98 reduces endothelial dysfunction by protecting blood-brain barrier (BBB) and improves neurological outcomes in mouse ischemia/reperfusion stroke model. J. Cereb. Blood Flow Metab. 40, 1953-1965 (2019).

19. Keasey, M. P., Scott, H. L., Bantounas, I., Uney, J. B. \& Kelly, S. MiR-132 is upregulated by ischemic preconditioning of cultured hippocampal neurons and protects them from subsequent OGD toxicity. J. Mol. Neurosci. 59, 404-410 (2016).

20. Colonna, M. \& Butovsky, O. Microglia function in the central nervous system during health and neurodegeneration. Annu. Rev. Immunol. 35, 441-468 (2017).

21. Ma, Y., Wang, J., Wang, Y. \& Yang, G. Y. The biphasic function of microglia in ischemic stroke. Prog. Neurobiol. 157, 247-272 (2017).

22. Otxoa-de-Amezaga, A. et al. Microglial cell loss after ischemic stroke favors brain neutrophil accumulation. Acta Neuropathol. 137, 321-341 (2019).

23. Kronenberg, G. et al. Distinguishing features of microglia- and monocytederived macrophages after stroke. Acta Neuropathol. 135, 551-568 (2018).

24. Sierra, A., Abiega, O., Shahraz, A. \& Neumann, H. Janus-faced microglia: beneficial and detrimental consequences of microglial phagocytosis. Front. Cell Neurosci. 7, 6 (2013).

25. Pluvinage, J. V. et al. CD22 blockade restores homeostatic microglial phagocytosis in ageing brains. Nature 568, 187-192 (2019).

26. Fuhrmann, M. et al. Microglial Cx3cr1 knockout prevents neuron loss in a mouse model of Alzheimer's disease. Nat. Neurosci. 13, 411-413 (2010).

27. Neher, J. J. et al. Inhibition of microglial phagocytosis is sufficient to prevent inflammatory neuronal death. J. Immun 186, 4973-4983 (2011).

28. Brown, G. C. \& Neher, J. J. Microglial phagocytosis of live neurons. Nat. Rev. Neurosci. 15, 209-216 (2014).

29. Neher, J. J. et al. Phagocytosis executes delayed neuronal death after focal brain ischemia. Proc. Natl. Acad. Sci. USA 110, E4098-E4107 (2013).

30. Alawieh, A., Langley, E. F. \& Tomlinson, S. Targeted complement inhibition salvages stressed neurons and inhibits neuroinflammation after stroke in mice. Sci. Transl. Med. 10, eaao6459 (2018).

31. Fricker, M. et al. MFG-E8 mediates primary phagocytosis of viable neurons during neuroinflammationJ. Neurosci.: Off. J. Soc. Neurosci. 32, 2657-2666 (2012).

32. Trajkovic, K. et al. Ceramide triggers budding of exosome vesicles into multivesicular endosomes. Science 319, 1244-1247 (2008).

33. Men, Y. et al. Exosome reporter mice reveal the involvement of exosomes in mediating neuron to astroglia communication in the CNS. Nat. Commun. 10, 4136 (2019)

34. Zhang, Y. et al. An RNA-sequencing transcriptome and splicing database of glia, neurons, and vascular cells of the cerebral cortex. J. Neurosci. 34 11929-11947 (2014).

35. Bellizzi, M. J., Geathers, J. S., Allan, K. C. \& Gelbard, H. A. Platelet-activating factor receptors mediate excitatory postsynaptic hippocampal injury in experimental autoimmune encephalomyelitis. J. Neurosci. 36, 1336-1346 (2016).

36. Ferracini, M., Rios, F. J., Pecenin, M. \& Jancar, S. Clearance of apoptotic cells by macrophages induces regulatory phenotype and involves stimulation of CD36 and platelet-activating factor receptor. Mediators Inflamm. 2013, 950273 (2013).

37. Koizumi, S., Hirayama, Y. \& Morizawa, Y. M. New roles of reactive astrocytes in the brain; an organizer of cerebral ischemia. Neurochemistry Int. 119, 107-114 (2018).

38. Brown, G. C. \& Neher, J. J. Inflammatory neurodegeneration and mechanisms of microglial killing of neurons. Mol. Neurobiol. 41, 242-247 (2010).
39. Lee, H. R. et al. The poly-gamma-d-glutamic acid capsule surrogate of the Bacillus anthracis capsule induces nitric oxide production via the platelet activating factor receptor signaling pathway. Mol. Immunol. 68, 244-252 (2015).

40. Tiedt, S. et al. RNA-Seq identifies circulating miR-125a-5p, miR-125b-5p, and miR-143-3p as potential biomarkers for acute ischemic stroke. Circ. Res. 121, 970-980 (2017)

41. Li, G. et al. Impact of microRNAs on ischemic stroke: from pre- to post-disease. Prog. Neurobiol. 163-164, 59-78 (2018).

42. Li, Q., Li, X., Wang, L., Zhang, Y. \& Chen, L. miR-98-5p acts as a target for Alzheimer's disease by regulating $A$ beta production through modulating SNX6 expression. J. Mol. Neurosci. 60, 413-420 (2016).

43. Cheng, J. et al. RNA N(6)-methyladenosine modification is required for miR-98/ MYCN axis-mediated inhibition of neuroblastoma progression. Sci. Rep. 10, 13624 (2020)

44. $\mathrm{Xu}, \mathrm{B}$. et al. Neurons secrete miR-132-containing exosomes to regulate brain vascular integrity. Cell Res. 27, 882-897 (2017).

45. Simeoli, R. \& Montague, K. Exosomal cargo including microRNA regulates sensory neuron to macrophage communication after nerve trauma. Nat. Commun. 8, 1778, (2017).

46. Sun, D. et al. Exosomes are endogenous nanoparticles that can deliver biological information between cells. Adv. Drug Deliv. Rev. 65, 342-347 (2013).

47. Morel, L. et al. Neuronal exosomal miRNA-dependent translational regulation of astroglial glutamate transporter GLT1. J. Biol. Chem 288, 7105-7116 (2013).

48. Janas, A. M., Sapoń, K., Janas, T., Stowell, M. H. B. \& Janas, T. Exosomes and other extracellular vesicles in neural cells and neurodegenerative diseases. Biochim. Biophys. Acta 1858, 1139-1151 (2016).

49. Anttila, J. E., Whitaker, K. W., Wires, E. S., Harvey, B. K. \& Airavaara, M. Role of microglia in ischemic focal stroke and recovery: focus on Toll-like receptors. Prog. Neuro-Psychopharmacol. Biol. Psychiatry 79, 3-14 (2017).

50. Chen, Z. \& Trapp, B. D. Microglia and neuroprotection. J. Neurochem. 136 10-17 (2016).

51. McDonald, J. S., Milosevic, D., Reddi, H. V., Grebe, S. K. \& Algeciras-Schimnich, A. Analysis of circulating microRNA: preanalytical and analytical challenges. Clin. Chem. 57, 833-840 (2011).

52. Hayakawa, K. et al. Transfer of mitochondria from astrocytes to neurons after stroke. Nature 535, 551-555 (2016).

53. Ito, $M$. et al. Brain regulatory $\mathrm{T}$ cells suppress astrogliosis and potentiate neurological recovery. Nature 565, 246-250 (2019).

54. Ashwal, S., Tone, B., Tian, H. R., Cole, D. J. \& Pearce, W. J. Core and penumbral nitric oxide synthase activity during cerebral ischemia and reperfusion. Stroke 29, 1037-1046 (1998)

55. Meng, H. L. et al. Neuronal soluble Fas ligand drives M1-microglia polarization after cerebral Ischemia. CNS Neurosci. Ther. 22, 771-781 (2016).

56. Thery, C., Amigorena, S., Raposo, G. \& Clayton, A. Isolation and characterization of exosomes from cell culture supernatants and biological fluids. Curr. Protoc. Cell Biol. Chapter 3, Unit 3.22 (2006).

57. $\mathrm{Xu}$, J. et al. Sequentially site-specific delivery of thrombolytics and neuroprotectant for enhanced treatment of ischemic stroke. ACS Nano 13, 8577-8588 (2019).

58. Tian, T. et al. Surface functionalized exosomes as targeted drug delivery vehicles for cerebral ischemia therapy. Biomaterials 150, 137-149 (2018).

59. Dong, Y. F. et al. Potential role of microRNA-7 in the anti-neuroinflammation effects of nicorandil in astrocytes induced by oxygen-glucose deprivation. J. Neuroinflamm. 13, 60 (2016).

60. $\mathrm{Ni}$, J. et al. MicroRNA let-7c-5p protects against cerebral ischemia injury via mechanisms involving the inhibition of microglia activation. Brain Behav. Immun. 49, 75-85 (2015). 